\title{
SALUD MENTAL, CAPITAL PSICOLÓGICO Y ADOPCIÓN DE PRÁCTICAS PREVENTIVAS ANTE LA COVID EN JÓVENES MENORES DE 30 AÑOS
}

MENTAL HEALTH, PSYCHOLOGICAL CAPITAL AND PREVENTIVE PRACTICES AGAINST COVID AMONG YOUNG ADULTS UNDER 30

\section{Marta Rodríguez Jiménez Departamento de Psicología, Universidad de Valladolid, Facultad de Educación (Palencia) marta.rodriguez.jimenez@gmail.com \\ Amalia Rodríguez-Rodríguez} Departamento de Psicología y Antropología, Universidad de Extremadura, Facultad de Educación (Badajoz)

Cómo citar este texto:

Rodríguez Jiménez, M. y Rodríguez-Rodríguez, A. (2022). Salud mental, capital psicológico y adopción de prácticas preventivas ante la covid en jóvenes menores de 30 años. Health and Addictions / Salud y Drogas, 22(1), 199-213. doi: 10.21134/haaj.v22i1.649 


\section{Resumen}

Introducción: Estudios previos han mostrado efectos negativos de las medidas de contención sobre la salud mental de jóvenes.

Objetivos: Por ello, en este trabajo nos proponemos estudiar factores relacionados con el desarrollo de problemas de salud mental en jóvenes españoles menores de 30 años y predictores de la adopción de prácticas preventivas. Metodología: Se diseño una encuesta online. La muestra estuvo formada por un total de 327 jóvenes. Los instrumentos utilizados fueron el General Health Questionnaire y la Escala de Capital Psicológico. Asimismo, se recogieron datos acerca de la adhesión a las siguientes medidas: distancia social, uso de guantes, mascarilla, higiene de manos y confinamiento.

Resultados: Los resultados mostraron un alto malestar. El 99.6\% presentó puntuaciones GHQ $\geq 3$, mientras que el $52.7 \%$ presentaron puntuaciones $\mathrm{GHQ} \geq 6$. Ser varón se asoció con peores niveles de salud mental y un menor cumplimiento del total de las medidas. Asimismo, un mayor capital psicológico se asoció con una mejor salud mental. Conclusiones: Los resultados apuntan a la necesidad de cuidar de la salud mental de los jóvenes durante la pandemia, especialmente de los varones. Es posible que, programas dirigidos a promocionar el capital psicológico, mejoren la salud mental durante la pandemia.

\section{Abstract}

Introduction: Previous studies have shown negative effects of COVID-19 contention measures on young adults' mental health.

Objectives: For this reason, factors associated to developing mental health problems in young adults under 30 and preventive practices predictors have been studied.

Methodology: An online survey has been designed. The sample was composed of 327 young adults. Data collection took place during the lockdown period. The measures used were: General Health Questionnaire and Psychological Capital Scale. Additionally, contention measures data about social distance, gloves use, mask use, hand hygiene and lockdown at home were collected.

Results: The results have shown high distress on study participants. $99.6 \%$ of the sample presented GHQ scores higher than 3 and $55.7 \%$ higher than 6 . To be a man was associated to poorer scores on mental health and lower compliance with preventive measures. Additionally, a higher psychological capital was associated to a better mental health.

Conclusions: The results have pointed out to the need of caring of young people' mental health during covid 19 pandemic. It is possible that programs addressed to promote psychological capital would improve mental health during the pandemic.

\section{Palabras clave}

Salud mental, jóvenes adultos, medidas de contención, covid

\section{Keywords}

Mental health, young adults, contention measures, covid 


\section{Introducción}

En enero del año 2020, los primeros casos de infección por un nuevo coronavirus, el SARS-CoV-2 fueron informados en España. Desde entonces, la pandemia de COVID ha cambiado la vida de millones de personas en todo el mundo. En la actualidad, a la espera de la vacunación efectiva de la población necesaria para frenar la expansión del virus, el control sobre la pandemia recae en la adopción de prácticas preventivas. Entre los estratos poblacionales, uno de los que mayor preocupación genera es la población de jóvenes. Los jóvenes tienden a encontrarse en fiestas masivas, a tener una intensa vida social y como parte del proceso de socialización, a rebelarse contra las medidas establecidas. Es por ello, que el estudio de los factores relacionados con el cumplimiento de las medidas en jóvenes cobra una importancia fundamental.

Estudios realizados hasta la fecha muestran que, comparados con personas de mayor edad, los más jóvenes se adhieren menos a la medida de distancia social (Kwok, et al. ,2020) y a las medidas en general (Tomczyk, Rahn \& Schmidt,2020; De La Vega, et al., 2020), además de practicar un menor número de medidas (Qeadan et al., 2020). En este sentido, un factor identificado como importante en la adopción de prácticas preventivas, es la percepción de riesgo personal (De la Vega et al., 2020; Marinthe, et al., 2020), y el riesgo de complicaciones graves es menor en la población joven. Asimismo, en línea con lo obtenido en la población general, la adherencia en jóvenes es mayor en el género femenino (Von Soest, et al., 2020; Nivette et al., 2020; Tomczyk et al., 2020) y menor en aquellos con patrones antisociales de conducta. En lo relativo al nivel educativo, Nivette et al. (2020) realizaron un estudio con 737 adultos jóvenes en Suiza y encontraron que existía una relación negativa entre el nivel de estudios y la adopción de las medidas.

\section{Salud mental durante la pandemia de COVID}

Una de las consecuencias no deseadas de la pandemia se encuentra en su impacto sobre la salud mental. Existen varios factores que contribuyen a este hecho: la imprevisibilidad de las consecuencias, a nivel social y sanitario; la novedad del hecho, que afortunadamente se está viendo atenuada; los cambios en el estilo de vida regular; las duras medidas que están teniendo que ser tomadas para contener el virus, que incluyen el aislamiento social y las cuarentenas o la dificultad de planificar el futuro (Vinkers et al., 2020).

Tal y como señalan Zhang W.R. et al. (2020) se espera que las consecuencias psicológicas de la pandemia en los más jóvenes sean mayores debido a que son más vulnerables al estrés. Además, se encuentran en un momento del ciclo vital en el que su situación externa es inestable, en lo referente a su carrera y a su trabajo (Alonzi, La Torre, \& Silver, 2020). En esta línea, estudios previos han mostrado que los jóvenes se encuentran preocupados por el impacto que la pandemia pueda tener en su trayectoria académica o profesional (Hawke et al., 2020) y factores como el desempleo (Achdut \& Refaeli, 2020) o la inseguridad laboral (Ganson, et al. ,2020) han sido ligados al desarrollo de problemas de salud mental durante la pandemia en esta población. Adicionalmente, se encuentran en una etapa del desarrollo en la cual las relaciones sociales cobran una importancia fundamental y las medidas de confinamiento y de distancia social podrían resultar especialmente duras para ellos (Akkaya-Kalayci et al., 2020).

Efectivamente, estudios en la población general han mostrado que los más jóvenes presentan mayores probabilidades de experimentar malestar psicológico durante la pandemia (Jia et al., 2020; Varma, et al., 2020). Por ejemplo, Fernández, et al. (2020) llevaron a cabo un trabajo en población argentina durante el confinamiento en este país. Los resultados mostraron que los más jóvenes presentaban un mayor riesgo de experimentar estrés. También Liu et al. (2020) encontraron altos niveles de depresión y ansiedad en una muestra de adultos jóvenes estadounidenses y Jia et al. (2020) hallaron que jóvenes durante el periodo de confinamiento presentaban una peor salud mental que personas más mayores.

Con referencia a los factores de riesgo y protectores encontrados hasta la fecha, la literatura científica avala que determinadas variables como ser mujer, conocer personas contagiadas, ser trabajador sanitario y padecer enfermedades previas aumentan los niveles de ansiedad (Wang, et al., 2020; Liu, et al., 2020; Kohlmann, et al., 2020) y por el contrario tener más de 40 años disminuye este riesgo. En lo relativo a trabajos en jóvenes, se ha encontrado que 
altos niveles de soledad y baja tolerancia al estrés se asocian con el desarrollo de problemas de ansiedad, depresión y TEP (Zhao, et al., 2020) y presentar problemas físicos o mentales previos se asocia con desarrollar problemas como angustia o depresión. En otro trabajo llevado a cabo por Alonzi et al. (2020), en una muestra de jóvenes de 18 a 35 años, se encontró que presentar problemas físicos y/o mentales previos se asociaba con desarrollar problemas de salud mental como angustia y depresión (Alonzi, et al.,2020) .

También la adherencia a medidas preventivas ha sido asociada a presentar problemas de salud mental. En este sentido, los escasos estudios realizados hasta la fecha muestran que cumplir las prácticas preventivas se asocia con menores niveles de depresión, ansiedad y estrés (Solomou \& Constantinidou, 2020; Zhang, C. et al., 2020). No obstante, aquellos que cumplen estrictamente las medidas de higiene, presentan mayor ansiedad (Zhang C. et al., 2020). Asimismo, se ha hallado una relación positiva entre presentar problemas de salud mental y la adherencia a las medidas (Qeadan et al., 2020). Por tanto, la relación entre salud mental y adherencia a las medidas podría depender de la existencia o no de un problema de salud mental, así como del tipo de problema involucrado. Es posible que, para aquellos sin un problema de salud mental, cumplir las prácticas preventivas se asocie a menores índices de depresión y ansiedad. No obstante, para aquellos que presentaran o hayan desarrollado problemas psicológicos durante la pandemia, esta relación podría ser positiva.

\section{Capital psicológico}

Es muy habitual en las investigaciones psicológicas la búsqueda del trauma, de los conflictos y sobre todo, centrarse en el déficit, frente a ello, surge un nuevo paradigma en investigación, la psicología positiva. Ésta se centra en la investigación de un concepto amplio de la salud, que abarque más componentes que se puedan aplicar para mejorar el bienestar y sobre todo, potenciar aquellos elementos que hagan la vida un poco más fácil al ser humano. Se basa en buscar herramientas, estrategias y planes que potencien entornos positivos, generen bienestar y se centra en ayudar al individuo en la búsqueda de la felicidad.

Entre los conceptos aportados por la Psicología Positiva se encuentra el concepto de capital psicológico. Este puede ser definido como un constructo psicológico de segundo orden que se refiere a una situación positiva para el desarrollo personal caracterizado por tener confianza en la resolución y poner el esfuerzo necesario para conseguir el éxito en tareas difíciles (autoeficacia); realizar atribuciones positivas sobre conseguir el éxito en el futuro (optimismo); perseverar en la obtención de metas, redirigiendo las vías para su obtención cuando es necesario (esperanza), y sostener la conducta a pesar de los obstáculos surgidos (resiliencia) (Avey, et al., 2011). El capital psicológico beneficia al individuo aumentando el bienestar psicológico, elevando el nivel de desempeño y productividad, por todo ello también mejora el ambiente psicológico, social y organizacional. Los individuos que tienen niveles elevados de capital psicológico esperan que sucedan más cosas buenas (optimismo), creen en su propio éxito (eficacia), lo vinculan a su esfuerzo y voluntad (esperanza) y son más resistentes ante la adversidad o la frustración ante los problemas (resiliencia) (Luthans, Youssef \& Avolio, 2007).

Trabajos realizados durante la pandemia muestran que el capital psicológico y especialmente, la resiliencia, funciona como amortiguador ante los problemas de salud mental (Li, Yang, Dou \& Cheung, 2020). Las personas resilientes tienen autonomía, habilidades para solucionar problemas y competencias sociales, desenvolviéndose mejor en ambientes poco favorables u hostiles (Luthans, et al., 2008). Asimismo, en el trabajo Liu et al. (2020) realizado con jóvenes durante la pandemia, se halló que la resiliencia se asociaba con menores niveles de ansiedad, mientras que el apoyo social por parte de familiares con menores índices de depresión.

\section{Objetivos}

Considerando la importancia de que los jóvenes adopten medidas de contención tanto para su propia salud como para frenar la expansión del SARS CoV-2, así como los posibles efectos adversos de la pandemia de COVID-19 sobre la salud mental, los objetivos de este trabajo se refieren a estudiar factores relacionados con la adopción de prácticas preventivas y con la salud mental de jóvenes menores de 30 años y conocer si el capital psicológico o alguno de sus factores funciona como factor protector en el transcurso de la pandemia. 
Rodríguez Jiménez, M. y Rodríguez-Rodríguez, A.

Se formularon las siguientes preguntas de investigación:

- ¿Con qué frecuencia se presentan problemas de salud mental en jóvenes durante la pandemia?

- ¿Cuáles son los predictores de la presencia de problemas de salud mental en jóvenes menores de 30 años durante la pandemia?

- ¿Funciona el capital psicológico o alguno de sus factores como protector contra el desarrollo de problemas de salud mental en jóvenes durante la pandemia?

- ¿Qué variables resultan predictivas de la adopción de prácticas preventivas contra la covid en jóvenes menores de 30 años?

\section{Metodología}

\section{Diseño}

Se trata de un estudio ex post facto y prospectivo, con muestreo en forma de bola de nieve.

\section{Participantes}

La muestra está formada por 327 personas, de las que 257 son mujeres (79.1\%). Pertenecen a los siguientes grupos de edad: entre 15 y 17 (11.3 \%) y entre 18 y 29 (89.7\%). En su mayoría están solteros (88.1\%) y presentan estudios superiores (56\%) o medios (27.2\%). La mayor parte son estudiantes (51.1\%) o trabajadores (21.4\%). El $70 \%$ de la muestra habita en núcleos urbanos. El $7.1 \%$ de los participantes informó de que presentaba patologías médicas que podrían suponer un riesgo ante la COVID y un $15.6 \%$ había acudido a un profesional de la salud mental en los últimos 12 meses. Al inicio de la investigación, todos los participantes fueron informados de los objetivos y se aseguró el anonimato y la confidencialidad de los datos. La participación en el estudio fue voluntaria y los participantes podían abandonar la encuesta en cualquier momento sin ningún tipo de repercusión.

\section{Instrumentos}

\section{Salud mental}

Para la estudiar la prevalencia de problemas de salud mental, se utilizó el General Health Questionnaire (Goldberg, 1992) en la versión de 12 ítems, un cuestionario validado en población española, por su alta fiabilidad, validez, sencillez de uso y amplia utilización, tanto en España como en terceros países. El GHQ-12 es un cuestionario autoadministrado, que mide diversos aspectos relacionados con el malestar psíquico (ansiedad, sentimientos depresivos, etc.). Es utilizado ampliamente como una prueba de screening, que permite clasificar a los individuos como con o sin problemas de salud mental. Un ejemplo de ítem es: “¿Se ha sentido capaz de tomar decisiones?". Si bien existen divergencias sobre el número de factores del instrumento, la mayor parte de los estudios muestran que los resultados reflejan un único factor general. El GHQ-12 posee dos métodos de corrección principales: la puntuación GHQ, que oscila entre 0 y 12 y que puntúa los ítems de acuerdo con un código binario (0-0-1-1) y una puntuación conforme a una escala tipo Likert entre 0 y 3 . En este trabajo, se ha utilizado la puntuación $\mathrm{GHQ}$, ya que se considera más adecuada cuando es utilizado para diagnosticar casos. Para este trabajo, el punto de corte se estableció en 6 , la mediana de la muestra. El alpha de Cronbach del instrumento en la muestra del estudio es .897. 


\section{Capital psicológico}

Para medir el capital psicológico, se utilizó la Escala de Capital Psicológico, de Omar et al., (2014), en versión para población general. La escala evalúa los cuatro factores del capital psicológico: la esperanza, el optimismo, la resiliencia y la autoeficacia. Cada ítem se valora en una escala tipo Likert de 0 a 3. Un ejemplo de ítem es: "Cuando tengo una dificultad, por lo general encuentro una salida" (resiliencia). El alpha de Cronbach de la escala total en la muestra es .891, mientras que para el factor resilencia es .740, para el optimismo .656, para la autoeficacia .795, y para la esperanza .799 .

\section{Variables sociodemográficas y medidas de contención}

Al principio de la encuesta, se recogieron datos acerca de la edad, género, nivel educativo, número de habitantes de la ciudad en la que se estaba viviendo el confinamiento, estado civil y situación laboral. Asimismo, se preguntó a los participantes si presentaban patologías médicas que pudieran suponer un riesgo ante la covid, y si habían acudido a un profesional de la salud mental en los últimos 12 meses. También con qué personas o personas estaban conviviendo durante el periodo de confinamiento. Para recabar datos acerca de las prácticas preventivas, se pidió a los participantes que valoraran en una escala de 0 (Nunca) a 4 (Siempre) su adherencia a cada una de las siguientes medidas: uso de guantes, higiene de manos, uso de la mascarilla, confinamiento y distancia social. Exactamente, se les preguntó en qué medida cumplían las medidas sanitarias. El cumplimiento total de las medidas se obtuvo sumando las puntuaciones del cumplimiento de cada medida.

\section{Procedimiento}

El procedimiento de muestreo fue en forma de bola de nieve. Para la recogida de datos, se diseñó una encuesta en Microsoft Form, que se distribuyó mediante medios electrónicos (email y whatsapp). La recogida de datos tuvo lugar durante el periodo de confinamiento (abril de 2020). Al inicio de la encuesta, se informó a los participantes de los objetivos de la investigación y se aseguró el anonimato y la confidencialidad de los datos. Los datos fueron descargados de Microsoft Form en una hoja de Excel y se volcaron sobre una base de datos en el paquete estadístico SPSS, versión 26, donde fueron analizados. Para el análisis de datos se utilizaron pruebas paramétricas cuando fue posible y cuando no, pruebas no paramétricas. Asimismo, se ha añadido la d de Cohen para el estudio del tamaño del efecto en la comparación entre grupos. Como valores de referencia, se han utilizado los recomendados por el propio Cohen (1988): una $d=0.2$, se considera un tamaño del efecto pequeño, una $d=0.5$ se refiere a un tamaño del efecto medio y una d igual o superior a 0.8 , una magnitud del efecto grande.

\section{Consideraciones éticas}

El estudio ha sido llevado a cabo siguiendo los procedimientos señalados en la Declaración de Helsinki. Se respetaron todos los derechos de privacidad de los participantes. La recogida de datos fue realizada de manera anónima. Los participantes fueron informados de los objetivos de la investigación antes del inicio de esta y de su derecho a rehusar participar, así como a abandonar la encuesta en el momento que lo desearan.

\section{Resultados}

\section{Frecuencia de problemas psicológicos en la muestra}

El 99.6\% de los participantes igualan o superan el umbral de 3, mientras que el $52.7 \%$ presentan puntuaciones iguales o superiores a 6 , de los que el $72.1 \%$ son mujeres y el $9.3 \%$ tienen entre 15 y 18 años. El $12.3 \%$ está casado. El $57.4 \%$ presenta estudios superiores y el $27.5 \%$ estudios medios. 
Rodríguez Jiménez, M. y Rodríguez-Rodríguez, A.

Tabla 1. Regresiones logísticas binaria sobre la salud mental

\begin{tabular}{|c|c|c|c|c|}
\hline Punto de corte & & $Q \geq 6$ & & \\
\hline Variable & OR & IC (95\%) & $p$ & $B$ \\
\hline \multicolumn{5}{|l|}{ Género } \\
\hline Hombre & 3,166 & $1,648-6,083$ & ,001 & 1,152 \\
\hline Mujer & REF & & & \\
\hline \multicolumn{5}{|l|}{ Edad } \\
\hline Entre 15 y 18 & REF & & & \\
\hline Mayor de 18 & .599 & 301-1,192 & 144 &,- 512 \\
\hline \multicolumn{5}{|l|}{ Situación laboral } \\
\hline Desemple. & ,926 & $0,414-2,072$ & 851 &,- 077 \\
\hline ERTE & 667 & $0,309-1,438$ & 301 &,- 405 \\
\hline Autónomo & ,278 & ,047-1,625 & 155 & $-1,281$ \\
\hline Trabajador & REF & & & \\
\hline Estudiante & 1,017 & $0,568-1,821$ & ,955 & ,017 \\
\hline \multicolumn{5}{|l|}{ Nivel educativo } \\
\hline Superior & REF & & & \\
\hline Medio & ,957 & $0,566-1,619$ & 871 &,- 044 \\
\hline Primarios o secundarios & ,729 & $0,395-1,344$ & 311 &,- 317 \\
\hline \multicolumn{5}{|l|}{ Profesional salud mental } \\
\hline Sí & REF & & & \\
\hline No & ,580 & ,299-1,122 & 106 &,- 545 \\
\hline \multicolumn{5}{|l|}{ Patologías médicas } \\
\hline Sí & REF & & & \\
\hline No & ,715 & ,286-1,791 & ,474 &,- 335 \\
\hline \multicolumn{5}{|l|}{ Confinamiento solitario } \\
\hline Sí & REF & & & \\
\hline No & 1,669 & ,232-12,005 & 611 & ,512 \\
\hline
\end{tabular}

En su mayor parte son estudiantes (52.9\%) o trabajadores (22.1\%). Un $7.8 \%$ presenta patologías médicas previas con riesgo ante la COVID y un $18.1 \%$ había acudido a un profesional de la salud mental en los últimos 12 meses. Los síntomas informados con mayor frecuencia fueron: pensar que se es una persona que no vale para nada (83.8 \%) y perder confianza en uno mismo (76.5\%).

Para conocer las variables relacionados con el desarrollo de problemas de salud mental, se realizó una regresión logística binaria, clasificando a los sujetos en dos grupos: aquellos con puntuaciones GHQ $\geq 6$ y aquellos con puntuaciones inferiores. Se observa que, comparados con las mujeres, los hombres presentan 3.166 veces más probabilidades de presentar problemas de salud mental (Tabla 1). El resto de variables no se relacionaron significativamente (Tabla 1).

\section{Capital psicológico}

La media de la muestra en la escala total es $44,27(8,70)$. Para conocer si existen diferencias significativas entre hombres y mujeres se realizó la t de Student, ya que se cumplen los supuestos. No existen diferencias significativas entre hombres y mujeres en capital total $(t=1,033 ; p=.305)$. El tamaño del efecto es -0.22 . Debido a que no se cumplen los supuestos, en el resto de factores se realizó la U de Mann Whitney. No existen diferencias significativas en resiliencia 
$(U=7219,5: p=.116)(d$ de cohen=0); optimismo $(U=8095 ; p=.666)(d$ de cohen=0); autoeficacia $(U=8028 ; p=.367)(d$ de cohen=0), ni esperanza $(U=3685 ; p=.960)(d=0)$. Tampoco existen diferencias significativas en el total $(U=2163$; $p=.60)(d=-0.125)$, ni en los factores de resiliencia $(U=4592,5 ; p=.348)(d=0)$, optimismo $(U=5037,5 ; p=, 657)(d=0)$, autoeficacia $(U=4990 ; p=, 547)(d=0.5)$ o esperanza $(U=2402,5 ; p=, 657)(d=0)$, dependiendo de la edad.

Para conocer la relación entre capital psicológico y salud mental, se realizó una regresión lineal, utilizando la corrección tipo Likert del GHQ. Los residuos siguen una distribución normal ( $\mathrm{k}-\mathrm{s}=.20)$ y no se observa heterocedasticidad. Se observa que presentar un mayor capital psicológico predice presentar una menor puntuación en GHQ ( $B=-, 359$; $\mathrm{p}=.00)$. El modelo explica el $16.5 \%\left(\mathrm{R}^{2}\right.$ ajustada=.165) de la varianza.

\section{Cumplimiento de medidas}

En la Tabla 2, pueden observarse los porcentajes de cumplimiento de cada medida individualmente y el número de medidas cumplidas. Para conocer qué variables se relacionan con el cumplimiento de cada medida, se realizó una regresión logística binaria, formando dos grupos: aquellos que cumplían siempre o casi siempre cada una de las medidas (higiene de manos, guantes, mascarilla, confinamiento y distancia social), y aquellos que no.

Tabla 2. Porcentaje de cumplimiento de cada medida y número de medidas cumplidas

\begin{tabular}{llllll}
\hline Medida & Nunca & Es raro & A veces & Casi siempre & Siempre \\
\hline Confinamiento & $0.3 \%$ & $1.2 \%$ & $2.8 \%$ & $22.2 \%$ & $73.5 \%$ \\
Distancia & $1.5 \%$ & $1.8 \%$ & $10.8 \%$ & $28 \%$ & $57.8 \%$ \\
Mascarilla & $36.3 \%$ & $8.3 \%$ & $17.8 \%$ & $16 \%$ & $21.5 \%$ \\
Lavado de manos & $0 \%$ & $3.4 \%$ & $15.6 \%$ & $51.5 \%$ & $29.4 \%$ \\
Guantes & $23.9 \%$ & $11 \%$ & $20.2 \%$ & $21.1 \%$ & $23.9 \%$ \\
Número de medidas & Una & Dos & Tres & Cuatro & Cinco \\
& $0.3 \%$ & $2.5 \%$ & $18.5 \%$ & $34.5 \%$ & $18.2 \%$ \\
\hline
\end{tabular}

Tal y como se observa en la Tabla 3, los estudiantes presentan menor probabilidad de no adoptar las medidas de confinamiento, mascarilla y guantes; los menores de 18 presentan mayor probabilidad de no cumplir la medida de distancia social y aquellos que han acudido a un profesional de la salud mental es menos probable que no cumplan la medida de higiene de manos. Asimismo, en la Tabla 4 se presenta el modelo ajustado por edad y género.

Tabla 3. Regresiones logísticas de variables sociodemográficas sobre el cumplimiento de cada medida.

\begin{tabular}{|c|c|c|c|c|c|c|c|c|c|c|c|c|}
\hline \multirow{2}{*}{$\frac{\text { MEDIDA }}{\text { Variable }}$} & \multirow[b]{2}{*}{$\%$} & \multicolumn{2}{|l|}{ Lavado } & \multicolumn{2}{|l|}{ Confin. } & \multicolumn{2}{|l|}{ Distancia } & \multicolumn{2}{|l|}{ Mascarilla } & \multicolumn{3}{|c|}{ Guantes } \\
\hline & & OR IC (95\%) & $p$ & OR IC (95\%) & $p$ & OR IC (95\%) & $p$ & OR IC (95\%) & $p$ & OR & IC (95\%) & $p$ \\
\hline \multicolumn{13}{|l|}{ Género } \\
\hline Hombre & & $1.405(0.737-2.677)$ & .30 & $1.556(0.472-5.124)$ & .46 & $1.213(.580-2.536)$ & .60 & $1.444(.814-2.560)$ & .20 & 1.393 & $.806-2.407$ & .235 \\
\hline Mujer & & REF & & REF & & REF & & REF & & REF & & \\
\hline \multicolumn{13}{|l|}{ Edad } \\
\hline 15 a 17 & 11.3 & $.805(0.320-2.024)$ & .64 & $.00 \quad .00$ & .99 & $2.593(1.159-5.800)$ & .02 & $1.482(.705-3.119)$ & .30 & 1.815 & $.878-3.750$ & .108 \\
\hline Más de 18 & 88.7 & REF & REF & & & REF & & REF & & & & \\
\hline \multicolumn{13}{|l|}{ Sit laboral } \\
\hline Estudiante & 51.1 & $1.843(0.862-3.938)$ & .11 & $0.193(0.047-.796)$ & .02 & $1.122(.510-2.472)$ & .77 & $\begin{array}{l}2.135(1.204- \\
3.786)\end{array}$ & .00 & 1.960 & $\begin{array}{l}1.114- \\
3.449\end{array}$ & .020 \\
\hline Desemple. & 12.2 & $1.500(0.539-4.176)$ & .43 & $0.269(0.031-2.321)$ & .23 & $1.059(.354-3.168)$ & .91 & $1.912(.856-4.268)$ & .11 & .972 & $.445-2.121$ & .942 \\
\hline ERTE & 13.5 & $0.600(0.176-2.046)$ & .41 & 0.787 (0.186-3.329) & .74 & .439 (.114-1.693) & .23 & 1.635 (.758-3.527) & .21 & 1,301 & ,611-2,769 & ,495 \\
\hline
\end{tabular}


Rodríguez Jiménez, M. y Rodríguez-Rodríguez, A.

\begin{tabular}{|c|c|c|c|c|c|c|c|c|c|c|c|c|}
\hline Autónomo & 1.8 & $\begin{array}{l}1.200(0.127- \\
11.374)\end{array}$ & .87 & $\begin{array}{l}2.100(0.210- \\
21.041)\end{array}$ & .52 & $1.200(.127-11.374)$ & .87 & $\begin{array}{l}2.059(.354- \\
11.988)\end{array}$ & .42 & 1.301 & $.611-2.769$ & .495 \\
\hline \multicolumn{13}{|l|}{ Trabajador } \\
\hline \multicolumn{13}{|l|}{ Nivel educ } \\
\hline Superior & 56.0 & REF & & REF & & REF & & REF & & REF & & \\
\hline Medio & 27.2 & $1.012(0.531-1.929)$ & .97. & $2.530(0.751-8.529)$ & .13 & $2.530(.751-8.529)$ & .13 & $1.327(.775-2.275)$ & .30 & 1.169 & $.699-1.955$ & .551 \\
\hline Menor & 16.9 & $0.940(0.432-2.046)$ & .87 & $2.019(0.467-8.733)$ & .34 & $2.019(.467-8.733)$ & .34 & $.774(.419-1.431)$ & .41 & .662 & $.361-1.213$ & .182 \\
\hline \multicolumn{13}{|l|}{ S. mental } \\
\hline Sí & 15.6 & REF & & REF & & & & & & & & \\
\hline No & & $\begin{array}{l}0.391(0.201- \\
0.760)^{*}\end{array}$ & .00 & $\begin{array}{l}2.382(0.304- \\
18.632)\end{array}$ & .40 & $2.382(.304-18.632)$ & .40 & $.582(.300-1.126)$ & .10 & .833 & $.455-1.527$ & .555 \\
\hline \multicolumn{13}{|l|}{ Pat. Méd. } \\
\hline Sí & 7.1 & REF & & REF & & & & & & & & \\
\hline No & & $0.509(0.200-1.296)$ & .15 & $0.997(0.125-7.974)$ & .99 & $.997(.125-7.974)$ & .99 & $.563(.216-1.469)$ & .24 & .769 & $.323-1.830$ & .552 \\
\hline
\end{tabular}

Tabla 4. Regresiones logísticas sobre el cumplimiento de cada medida, ajustadas por edad y género

\begin{tabular}{|c|c|c|c|c|c|c|c|c|c|c|c|}
\hline MEDIDA & & Lavado & & Confin. & & Distancia & & Mascarilla & & Guantes & \\
\hline Variable & $\%$ & OR IC (95\%) & $p$ & OR IC (95\%) & $p$ & OR IC (95\%) & $p$ & $\begin{array}{l}\text { OR } \\
\text { IC (95\%) }\end{array}$ & $p$ & IC (95\%) & $p$ \\
\hline \multicolumn{12}{|l|}{ Sit laboral } \\
\hline Estudiante & 51.1 & $2,183(, 997-4,777)$ & .051 & ,200 (,048-,831) & .027 & ,912(,392-2,125) & .83 & $2,210(1,208-4,045)$ & .01 & $1,996(1,103-3,613)$ & .022 \\
\hline Desemple. & 12.2 & $1,635(, 582-4,597)$ & .35 &, $278(, 032-2,421)$ & .24 & $1,030(, 340-3,121)$ & .95 & $2,049(, 908-4,622)$ & .08 & $1,017(, 462-2,239)$ & .96 \\
\hline ERTE & 13.5 & ,635 (,185-2,179) & .47 & ,812 (,190-3,470) & .77 & ,452(,117-1,752) & .25 & $1,893(, 321-11,170)$ & .48 & $1,381(, 644-2,961)$ & .40 \\
\hline Autónomo & 1.8 & $1,110(, 116-10,621)$ & .92 & $2,001(, 196-20,377)$ & .55 & $1,152(, 121-10,993)$ & .91 & $1,759(, 808-3,828)$ & .15 & $1,095(, 204-5,878)$ & .91 \\
\hline Trabajador & 21.4 & REF & & REF & & REF & & REF & & REF & \\
\hline Edad & & ,571 (,220-1,484) & .25 & NC & & $2,493(1,040-5,977)$ & .04 & $1,191(, 541-2,618)$ & .66 & $1,373(, 636-2,961)$ & .41 \\
\hline Género & & ,680 (,350-1,319) & .25 & $1,260(, 366-4,345)$ & .71 & $1,225(, 575-2,610)$ & .60 & $1,611(, 892-2,910)$ & .11 & $1,506(, 856-2,650)$ & .15 \\
\hline \multicolumn{12}{|l|}{ Nivel educ } \\
\hline Superior & 56.0 & REF & & REF & & REF & & REF & & REF & \\
\hline Medio & 27.2 & $1,042(, 530-2,050)$ & .90 & $2,491(, 738-8,409)$ & .13 & $2.530(.751-8.529)$ & .36 & $1,182(, 671-2,082)$ & .56 & $0,951(, 553-1,635)$ & .04 \\
\hline Menor & 16.9 & $1,009(, 440-2,314)$ & .98 & $2,050(, 472-8,896)$ & .33 & ,908 (,399-2,067) & .81 & ,671(,345-1,306) & .24 & $0,502(, 258-, 977)$ & .85 \\
\hline Edad & & ,801 (,294-2,184) & .66 & $\mathrm{NC}$ & & $2,335(, 924-5,904)$ & .07 & $1,651(, 727-3,750)$ & .23 & $2,354(1,054-5,261)$ & .03 \\
\hline Género & & ,717 (,376-1,369) & .31 & $1,569(, 472-5,210)$ & .46 & $1,285(, 608-2,717)$ & .51 & $1,435(, 807-2,552)$ & .21 & $1,391(, 801-2,416)$ & .24 \\
\hline S. mental & 15.6 & ,387(,198--,757) & .00 & $2,374(, 303-18,604)$ & .41 & $1,490(, 554-4,006)$ & .43 & ,593(,304-1,155) & .12 & ,779(,420-1,445) & .42 \\
\hline Edad & & ,894 (,352-2,273) & .81 & $\mathrm{NC}$ & & $\begin{array}{l}2,5378(1,128- \\
5,706)\end{array}$ & .02 & $1,573(, 743-3,326)$ & .23 & $1,862(, 897-3,865)$ & .09 \\
\hline Género & & $1,368(, 711-2,635)$ & .34 & $1,577(, 478-5,205)$ & .45 & $2.382(.304-18.632)$ & .40 & $1,449(, 815-2,578)$ & .20 & $1,414(, 815-2,450)$ & .21 \\
\hline Pat. Méd. & 7.1 & $0.511(2,00-1,308)$ & .16 & ,985 (,123-7,901) & .98 & $3,553(, 465-27,172)$ & .22 &, $536(, 205-1,406)$ & .20 & ,734(,306-1,756) & .48 \\
\hline Edad & & ,843 (,334-2,130) & .71 & NC & & $2,499(1,111-5,618)$ & .02 & $1,566(, 741-3,310)$ & .24 & $1,867(, 900-3,876)$ & .09 \\
\hline Género & & $1,411(0,738-2,698)$ & .298 & $1,550(, 470-5,107)$ & .47 & $1,244(, 589-2,626)$ & .56 & $1,492(, 839-2,653)$ & .17 & $1,442(, 832-2,500)$ & .19 \\
\hline
\end{tabular}

\section{Cumplimiento de todas las medidas}

Con referencia al cumplimiento total, para conocer si existen diferencias significativas entre hombres y mujeres, se realizó la U de Mann Whitney, ya que no se cumplen los supuestos para pruebas paramétricas. Tal y como se observa en la Fig. 1, las mujeres adoptan más las medidas. Además, estas diferencias resultan significativas $(U=6433,500$; $p=.003)(d=0.66)$. Asimismo, para conocer si existen diferencias entre grupos educativos, se realizó la prueba de Kruskal Wallis, ya que no se cumplen los supuestos para el ANOVA. No existen diferencias significativas entre los 
grupos en función del nivel educativo $(\mathrm{H}(2)=, 887 ; \mathrm{p}=.642)$. Para conocer si existen diferencias en función de la situación laboral, se procedió de la misma forma. De nuevo, no existen diferencias entre los grupos en función de la situación laboral $(\mathrm{H}(3)=3,622 ; p=, 305)$. Tampoco se cumple el supuesto de normalidad en el caso de la edad. Por ello, se realizó la prueba $U$. Las diferencias no resultan significativas $(U=4380,0 ; p=, 111)(d=0.28)$.

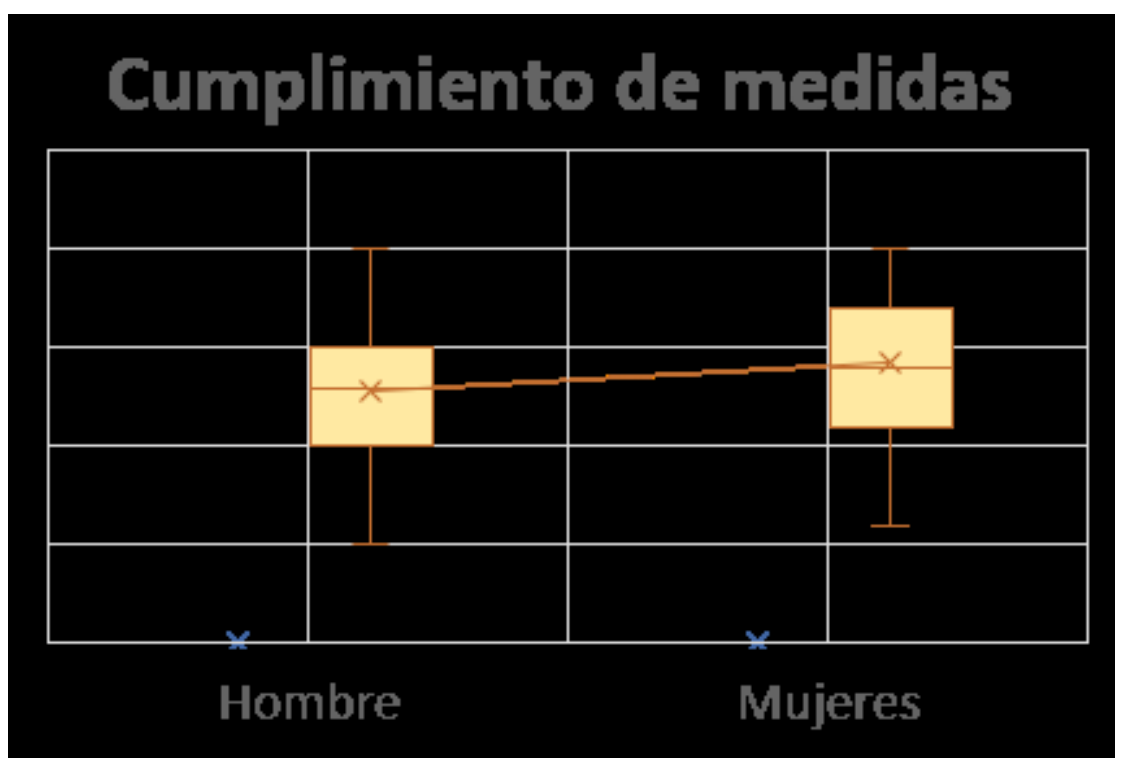

Fig 1. Diferencias de género en el cumplimiento total de las medidas

Discusión

Los objetivos de este trabajo se referían a estudiar la adopción de medidas preventivas ante la covid y la salud mental de jóvenes menores de 30 años durante la pandemia de COVID-19.

En primer lugar y en relación con la adopción de prácticas preventivas, los resultados en general muestran un alto cumplimiento de las medidas obligatorias o muy recomendadas en el momento de realización de la encuesta: confinamiento, distancia de seguridad e higiene de manos. No obstante, es reseñable que únicamente el $66.7 \%$ de la muestra afirma cumplir siempre o casi siempre estas tres medidas conjuntamente. En línea con lo esperable, la adopción de medidas no recomendadas oficialmente resulta menor. Con referencia al número de medidas adoptadas, los resultados muestran que el $96.3 \%$ de los jóvenes ha adoptado al menos una medida, resultados muy similares a los obtenidos por Atchison et al. (2020), en el Reino Unido, quienes encontraron que el 93\% de adultos en el Reino Unido había adoptado al menos una medida.

En lo relativo a la adopción de cada medida individualmente, la medida más adoptada fue el confinamiento en domicilio. El confinamiento fue una medida generalizada y además, comparado con otras medidas, como la higiene de manos, su "violación" resulta más evidente. La vigilancia por parte de los Cuerpos y Fuerzas de Seguridad del Estado y por la ciudadanía en general, podrían dar cuenta, al menos, de una parte, del elevado cumplimiento observado, sumado a las campañas realizadas para concienciar a la población de su necesidad. Con referencia al uso de la mascarilla, en este trabajo solo el 21.5 \% manifestó usarla siempre. En este sentido, es necesario considerar que, en abril de 2020, el uso de la mascarilla no era una medida obligatoria y además, existían divergencias sobre su utilidad. Trabajos como el de Geana (2020) muestran que existe una relación entre la percepción de la utilidad de una medida y su adopción. La misma lógica podría ser aplicada a la utilización de guantes.

Con referencia a la distancia social, los jóvenes en este trabajo se adhieren más esta medida que en el trabajo de Von Soest et al. (2020), realizado con un grupo de adolescentes en Oslo. Es posible que la edad explique, al menos, una parte, de las diferencias observadas. En este aspecto, los participantes en este estudio son mayores y como se ha señalado anteriormente, se ha encontrado una relación entre presentar una mayor edad y la adopción de las 
medidas. De hecho, en consonancia con el trabajo de Von Soest et al. (2020), los menores de 18 años participantes en este estudio, presentan mayores probabilidades de no adoptar la medida de distancia social.

Respecto a los factores asociados con el cumplimiento total de las medidas, los resultados muestran que las mujeres cumplen significativamente más todas las medidas que los hombres, resultados consistentes con otros estudios realizados en el contexto de la pandemia en adultos (Kwok et al., 2020; Galasso, et al., 2020) y en jóvenes (Von Soest et al., 2020). Parece que ni factores ideológicos, ni socioeconómicos, explican las diferencias observadas y es la aversión al riesgo ante situaciones nuevas la causa más probable de las diferencias de género observadas (Chesser, et al., 2020). Otro de los resultados obtenidos en este trabajo se refiere a que los estudiantes utilizan con mayor probabilidad mascarilla y guantes, medidas no obligatorias cuando se realizó la encuesta. Es posible que se deba a las fuentes de información consultadas por los estudiantes. En este aspecto, trabajos como el de Chesser et al. (2020), ponen de manifiesto que los estudiantes utilizan preferentemente internet o las redes sociales para obtener información acerca de la COVID-19, medios alternativos a los tradicionales como la televisión o los periódicos, donde más fácilmente pueden entrar en contacto con información falsa, poco fiable o alternativa a la mostrada por las fuentes oficiales. También podría deberse a mayores niveles de alarma o de ansiedad. Sin embargo, los resultados en este trabajo no muestran que los estudiantes presenten con mayor probabilidad problemas de salud mental.

Los resultados también muestran que los jóvenes con problemas de salud mental cumplen con mayor probabilidad la medida de higiene de manos, en línea con lo encontrado por Solomou y Constantinidou (2020), que hallaron que aquellos con altos niveles de ansiedad cumplían más con las medidas de higiene. Parece lógico que aquellas personas preocupadas por la amenaza del virus, con altos niveles de ansiedad, acudan a lavarse las manos con mayor frecuencia para asegurarse de que no van a contagiarse. Por otra parte, obsesión con la higiene personal e infecciones por patógenos, es un síntoma de trastornos anteriormente categorizados como de ansiedad como el Trastorno Obsesivo Compulsivo (DSM-IV).

Con referencia al segundo objetivo, que se refería a estudiar la salud mental y factores relacionados con el desarrollo de problemas de salud mental en jóvenes menores de 30, los resultados muestran una alta prevalencia de malestar psicológico ya que el $\mathbf{9 9 . 6 \%}$ de la muestra supera el umbral de 3. Los resultados resultan superiores a los informados por Domínguez-Salas et al. (2020) en un estudio realizado en España en la población general de adultos en las mismas fechas. Es posible que la discordancia se deba a la edad de los participantes ya que, como ha sido señalado anteriormente, estudios previos realizados durante la pandemia de COVID han mostrado que los jóvenes presentan una peor salud mental (Fernández, et al., 2020). En este sentido, tal y como señalan Zhao et al. (2020) los jóvenes presentan una mayor vulnerabilidad al estrés que los adultos y se encuentran en una etapa del ciclo vital de inestabilidad en lo referente a su trabajo y/o a sus estudios.

Respecto a los factores de riesgo encontrados, los resultados muestran que los hombres presentan puntuaciones $\mathrm{GHQ} \geq 6$ con mayor probabilidad. Estos resultados no coinciden con trabajos previos realizados en el contexto de la pandemia como los llevados a cabo por Solomou y Constantinidou (2020) y en general, considerando el tipo de problema que mide el GHQ, resultan sorprendentes, ya que la prevalencia de los trastornos depresivos es mayor en las mujeres, así como la de trastornos de ansiedad (OMS, 2017). Es posible que estos resultados se deban a que en este trabajo la proporción de hombres que trabajan es mayor que la proporción de mujeres. Factores como miedo al desempleo por la crisis económica o la obligación de abandonar el domicilio pudieron incrementar el estrés de estos participantes. Asimismo, el número de varones con patologías psiquiátricas previas resulta mayor y estudios en el contexto de la pandemia muestran que esta población se encuentra en riesgo de recaídas (Alonzi, et al., 2020).

Por último, los resultados también muestran que presentar un mayor capital psicológico predice presentar menores problemas de salud mental. Estos resultados son consistentes con estudios previos que muestran que el capital psicológico funciona como factor protector ante el desarrollo de problemas afectivos en distintas poblaciones, entre ellas, la población de jóvenes universitarios (Selvaraj, 2015). En este sentido, las personas con mayor capital resultan más optimistas y presentan una mayor capacidad para hacer frente a situaciones adversas (Luthans, et al., 2007). Por ello, no resulta sorprendente que en un contexto adverso como la pandemia de COVID y en un periodo de confinamiento, las personas con mayor capital presenten menores niveles de estrés y con ello, menos síntomas asociados con problemas de salud mental. 
Como conclusión, los resultados de este trabajo muestran un alto cumplimiento de las medidas obligatorias durante el periodo de confinamiento y un menor cumplimiento de aquellas no recomendadas oficialmente. Las mujeres adoptaron más el conjunto de las medidas en consonancia con trabajos previos y los estudiantes usaron guantes y mascarilla con mayor probabilidad. Asimismo, aquellos con peor salud mental adoptaron significativamente más la medida de higiene de manos. Los resultados muestran también una pobre salud mental en los encuestados. Ser hombre se relacionó con una mayor probabilidad de presentar problemas de salud mental y presentar un mayor capital psicológico se relacionó con menores problemas, por lo podría ser interesante potenciar el capital psicológico de cara a futuras pandemias.

\section{Conflicto de intereses}

Ninguno.

\section{Financiación}

La presente investigación no ha recibido ayudas específicas provenientes de agencias del sector público, sector comercial o entidades sin ánimo de lucro.

\section{Referencias}

Achdut, N., \& Refaeli, T. (2020). Unemployment and psychological distress among young people during the COVID-19 pandemic: Psychological resources and risk factors. International Journal of Environmental Research and Public Health, 17(19), 7163 https://doi.org/10.3390/ijerph17197163

Akkaya-Kalayci, T., Kothgassner, O. D., Wenzel, T., Goreis, A., Chen, A., Ceri, V., \& Özlü-Erkilic, Z. (2020). The Impact of the COVID-19 Pandemic on Mental Health and Psychological Well-Being of Young People Living in Austria and Turkey: A Multicenter Study. International Journal of Environmental Research and Public Health, 17(23), 9111. https:// doi.org/10.3390/ijerph17239111

Alonzi, S., La Torre, A., \& Silverstein, M. W. (2020). The psychological impact of preexisting mental and physical health conditions during the COVID-19 pandemic. Psychological trauma: theory, research, practice, and policy, 12, S236-S238. https://doi.org/10.1037/tra0000840

Atchison, C., Bowman, L. R., Vrinten, C., Redd, R., Pristerà, P., Eaton, J., \& Ward, H. (2021). Early perceptions and behavioural responses during the COVID-19 pandemic: a cross-sectional survey of UK adults. BMJ open, 11(1), e043577 http://dx.doi.org/10.1136/bmjopen-2020-043577

Avey, J. B., Reichard, R. J., Luthans, F., \& Mhatre, K. H. (2011). Meta-analysis of the impact of positive psychological capital on employee attitudes, behaviors, and performance. Human resource development quarterly, 22(2), 127152. https://doi.org/10.1002/hrdq.20070

Chesser, A., Drassen Ham, A., \& Keene Woods, N. (2020). Assessment of COVID-19 knowledge among university students: implications for future risk communication strategies. Health Education \& Behavior, 47(4), 540-543. https:// doi.org/10.1177/1090198120931420

Cohen, J. (1988). Statistical Power Analysis for the Behavioral Sciences. New York, NY: Routledge Academic.

De La Vega, R., Ruíz-Barquín, R., Boros, S., \& Szabo, A. (2020). Could attitudes toward COVID-19 in Spain render men more vulnerable than women?. Global public health, 15(9), 1278-1291. https://doi.org/10.1080/17441692.2020.1 
Domínguez-Salas, S., Gómez-Salgado, J., Andrés-Villas, M., Díaz-Milanés, D., Romero-Martín, M., \& Ruiz-Frutos, C. (2020). Psycho-emotional approach to the psychological distress related to the COVID-19 pandemic in Spain: a cross-sectional observational study. Healthcare,8 (3),190. https://doi.org/10.3390/healthcare8030190

Fernández, R. S., Crivelli, L., Guimet, N. M., Allegri, R. F., \& Pedreira, M. E. (2020). Psychological distress associated with COVID-19 quarantine: Latent profile analysis, outcome prediction and mediation analysis. Journal of affective disorders, 277, 75-84. https://doi.org/10.1016/j.jad.2020.07.133.

Galasso, V., Pons, V., Profeta, P., Becher, M., Brouard, S., \& Foucault, M. (2020). Gender differences in COVID-19 attitudes and behavior: Panel evidence from eight countries. Proceedings of the National Academy of Sciences, 117(44), 27285-27291. https://doi.org/10.1073/pnas.2012520117

Ganson, K. T., Tsai, A. C., Weiser, S. D., Benabou, S. E., \& Nagata, J. M. (2020). Job insecurity and symptoms of anxiety and depression among US young adults during COVID-19. Journal of Adolescent Health, 68(1), 53-56. https://doi. org/10.1016/j.jadohealth.2020.10.008

Geana, M. V. (2020). Kansans in the middle of the pandemic: risk perception, knowledge, compliance with preventive measures, and primary sources of information about COVID-19. Kansas journal of medicine, 13, 160.

Goldberg D. (1992). General health questionnaire (GHQ-12). Windsor: NFER-Nelson.

Hawke, L. D., Barbic, S. P., Voineskos, A., Szatmari, P., Cleverley, K., Hayes, E., ... \& Henderson, J. L. (2020). Impacts of COVID-19 on Youth Mental Health, Substance Use, and Well-being: A Rapid Survey of Clinical and Community Samples, The Canadian Journal of Psychiatry, 65(10), 701-709. https://doi.org/10.1177/0706743720940562

Jia, R., Ayling, K., Chalder, T., Massey, A., Broadbent, E., Morling, J. R., ... \& Vedhara, K. (2020). Young people, mental health and COVID-19 infection: the canaries we put in the coal mine. Public health, 189, 158-161. https://doi. org/10.1016/j.puhe.2020.10.018

Kohlmann, S., Gierk,B., Hilbert, A., Brähler E., \& Löwe, B. (2016). La superposición de los síndromes somáticos, ansiosos y depresivos: un análisis poblacional. Revista de investigación psicosomática, 90, 51-56.

Kwok, K. O., Li, K. K., Chan, H. H., Yi, Y. Y., Tang, A., Wei, W. I., \& Wong, Y. S. (2020). Community responses during the early phase of the COVID-19 epidemic in Hong Kong: risk perception, information exposure and preventive measures. Emerging infectious diseases, 26, 1575-1579. https://doi.org/10.3201/eid2607.200500

Li, J.B., Yang, A., Dou, K. \& Cheung, R.Y. (2020). Self-Control Moderates the Association Between Perceived Severity of Coronavirus Disease 2019 (COVID-19) and Mental Health Problems Among the Chinese Public, International Journal of Environmental Research and Public Health, 17, 4820. https://doi.org/10.3390/ijerph17134820

Liu, C. H., Zhang, E., Wong, G. T. F., \& Hyun, S. (2020). Factors associated with depression, anxiety, and PTSD symptomatology during the COVID-19 pandemic: Clinical implications for US young adult mental health. Psychiatry research, 290, 113172. https://doi.org/10.1016/j.psychres.2020.113172

Luthans, F., Norman, S. M., Avolio, B. J., \& Avey, J. B. (2008). The mediating role of psychological capital in the supportive organizational climate-employee performance relationship. The International Journal of Industrial, Occupational and Organizational Psychology and Behavior, 29(2), 219-238 https://doi.org/10.1002/job.507 
Luthans, F., Youssef, C. M., \& Avolio, B. J. (2007). Psychological capital: Developing the human competitive edge. New York: Oxford.

Marinthe, G., Brown, G., Delouvée, S., \& Jolley, D. (2020). Looking out for myself: Exploring the relationship between conspiracy mentality, perceived personal risk, and COVID-19 prevention measures. British Journal of Health Psychology, 25(4), 957-980. https://doi.org/10.1111/bjhp.12449

Nivette, A., Ribeaud, D., Murray, A., Steinhoff, A., Bechtiger, L., Hepp, U., ... \& Eisner, M. (2020). Non-compliance with COVID-19-related public health measures among young adults in Switzerland: Insights from a longitudinal cohort study. Social Science \& Medicine, 268, 113370. https://doi.org/10.1016/j.socscimed.2020.113370

Omar, A., Salessi, S., \& Urteaga, F. (2014). Diseño y validación de la escala CapPsi para medir capital psicológico. Liberabit, 20(2), 315-323.

Organización Mundial de la Salud (2017). Depression and other common mental disorders: global health estimates (No. WHO/MSD/MER/2017.2).

Qeadan, F., Akofua Mensah, N., Tingey, B., Bern, R., Rees, T., Talboys, S., ... \& Shoaf, K. (2020). What protective health measures are Americans taking in response to COVID-19? Results from the COVID Impact Survey. International Journal of Environmental Research and Public Health, 17(17), 6295. https://doi.org/10.3390/ijerph17176295

Selvaraj, P. R. (2015). Using positive psychological capital to predict mental health in college students: Implications for counseling and higher education (Doctoral dissertation, Ohio University)

Solomou, I., \& Constantinidou, F. (2020). Prevalence and predictors of anxiety and depression symptoms during the COVID-19 pandemic and compliance with precautionary measures: Age and sex matter. International journal of environmental research and public health, 17(14), 4924. https://doi.org/10.3390/ijerph17144924

Tomczyk, S., Rahn, M., \& Schmidt, S. (2020). Social distancing and stigma: Association between compliance with behavioral recommendations, risk perception, and stigmatizing attitudes during the COVID-19 outbreak. Frontiers in psychology, 11, 1821. https://doi.org/10.3389/fpsyg.2020.01821

Varma, P., Junge, M., Meaklim, H., \& Jackson, M. L. (2020). Younger people are more vulnerable to stress, anxiety and depression during COVID-19 pandemic: A global cross-sectional survey. Progress in Neuro-Psychopharmacology and Biological Psychiatry, 110236. https://doi.org/10.1016/j.pnpbp.2020.110236

Vinkers, C. H., van Amelsvoort, T., Bisson, J. I., Branchi, I., Cryan, J. F., Domschke, K., ... \& van der Wee, N. J. (2020). Stress resilience during the coronavirus pandemic. European Neuropsychopharmacology, 35, 12-16. https://doi. org/10.1016/j.euroneuro.2020.05.003

Von Soest, T., Bakken, A., Pedersen, W., \& Sletten, M. A. (2020). Life satisfaction among adolescents before and during the COVID-19 pandemic. Tidsskrift for Den norske legeforening, 140, 10. https://doi.org/10.4045/tidsskr.20.0437

Wang, Y., Di, Y., Ye, J., \& Wei, W. (2021). Study on the public psychological states and its related factors during the outbreak of coronavirus disease 2019 (COVID-19) in some regions of China. Psychology, health \& medicine, 26(1), 13-22. https://doi.org/10.1080/13548506.2020.1746817

Zhang, C., Ye, M., Fu, Y., Yang, M., Luo, F., Yuan, J., \& Tao, Q. (2020). The psychological impact of the COVID-19 pandemic on teenagers in China. Journal of Adolescent Health, 67(6), 747-755. https://doi.org/10.1016/j. jadohealth.2020.08.026 
Rodríguez Jiménez, M. y Rodríguez-Rodríguez, A.

Zhang, W. R., Wang, K., Yin, L., Zhao, W. F., Xue, Q., Peng, M., ... \& Wang, H. X. (2020). Mental health and psychosocial problems of medical health workers during the COVID-19 epidemic in China. Psychotherapy and psychosomatics, 89(4), 242-250. https://doi.org/10.1159/000507639

Zhao, S. Z., Wong, J. Y. H., Wu, Y., Choi, E. P. H., Wang, M. P., \& Lam, T. H. (2020). Social distancing compliance under Covid-19 pandemic and mental health impacts: A population-based study. International journal of environmental research and public health, 17(18), 6692. https://doi.org/10.3390/ijerph17186692 